\title{
LETTER OPEN \\ Crystal structure of SARS-CoV-2 nsp10/nsp16 2'-O-methylase and its implication on antiviral drug design
}

Signal Transduction and Targeted Therapy (2020)5:131

\section{Dear Editor,}

The unexpected outbreak of a novel human coronavirus infection has imposed great threat to public health. Thus far, this newly-identified virus has spread to 215 countries and territories, infected more than 3.5 million people, and caused over 240,000 deaths worldwide. ${ }^{1}$ Although intensified countermeasures have been implemented globally to control the virus infection, the pandemic is still surging with a daily increase in infection case of over 65,000 ever since April 1st 2020 . $^{1}$ No prophylactic vaccines or clinical drugs are currently available to prevent or treat the disease, namely coronavirus disease 2019 (COVID-19).

Coronavirus is a group of enveloped positive-sensed RNA viruses that replicate in host cell cytoplasm via a large membraneassociated RNA replication/transcription machinery comprising at least sixteen virus-encoded non-structural proteins (nsp1 to nsp16). Among these, nsp10 and nsp16 form a protein complex, which functions to catalyze the methylation of the penultimate nucleotide of the viral RNA cap at the ribose 2'-O position. Such methylation process would convert the RNA cap of the virus from a cap-0 structure (featured as ${ }^{7 \mathrm{Me}} \mathrm{GpppA}$...) into a cap-1 structure

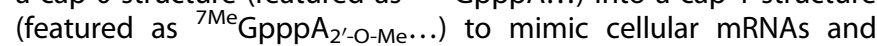
thereby to prevent recognition of viral RNAs by host innate immunity. ${ }^{2,3}$ In order to learn the structural features of SARS-CoV-2 nsp10/nsp16 2'-O-methylase, the two proteins were co-expressed in Escherichia coli (E. coli) and purified as a stable heterodimer complex in solution (Supplementary Fig. S1a). The protein complex was then crystallized in the presence of S-adenosyl-Lmethionine (SAM, a methyl donor for the methylation reaction) supplemented at a 1:5 protein-to-SAM molar ratio. The structure was solved at $2.5 \AA$ resolution. We also crystallized nsp10/nsp16 without additional SAM and solved its structure at $2.8 \AA$ resolution (Supplementary Table S1). In each case, the solved structure contains a single 1:1 bound nsp10/nsp16 hetero-complex in the crystallographic asymmetric unit. In addition, the electron densities for the SAM molecule were clearly observed in both structures (Supplementary Fig. S2a, b), demonstrating that the protein had captured SAM simultaneously when expressed in E. coli. As expected, the two structures solved in this study are quite similar to each other, showing a root mean square deviation (RMSD) of $\sim 0.2 \AA$ for 115 equivalent nsp10 Cas and of $\sim 0.2 \AA$ for 298 nsp16 Cas, respectively (Supplementary Fig. S2c).

Overall, the nsp10/nsp16 structure can be viewed as an nsp16 monomer perching on top of an nsp10 molecule (Fig. 1a). The topresiding nsp16 is composed of twelve $\beta$-strands, seven a-helices, and five $3_{10}$ helices (Supplementary Fig. S3a). These secondary structural elements assemble into a compact fold. The core of the structure is made of strands $\beta 1-\beta 7$ and helices $a Z, a A, a D$, and $a E$. This central core is further decorated at the protein $\mathrm{N}$-terminus by the $\eta 1310^{-}$-helix, $\beta 8$-strand, and af-helix, between strands $\beta 3$ and $\beta 4$ by the $\beta 9$-strand and $\eta 23_{10}$-helix, between strand $\beta 4$ and helix
; https://doi.org/10.1038/s41392-020-00241-4

$\mathrm{aD}$ by the $n 33_{10}$-helix, and at the C-terminus by strands $\beta 10-\beta 12$, a-helices ag and ah, and $3_{10}$-helices $\eta 4$ and $\eta 5$. The bottom-sitting nsp10 comprises three $\beta$-strands, three $\alpha$-helices and two $3_{10}$ helices (Supplementary Fig. S3b). Strands $\beta 1^{\prime}-\beta 3^{\prime}$ form a central $\beta$-sheet, and the helical components (a-helices $\alpha 2^{\prime}-\alpha 4^{\prime}$ and $3{ }_{10^{-}}$ helices $\eta 1^{\prime}$ and $\eta 2^{\prime}$ ) are packed together to cover one surface of the central sheet (Fig. 1a). Both the individual protein-subunit structure and the protein-subunit interaction mode of the SARSCoV-2 nsp10/nsp16 complex resemble those observed in other beta-coronaviruses. Superimposition of our structure onto those of SARS-CoV (PDB: 3R24) and MERS-CoV (PDB: 5YN5) revealed an RMSD of $\sim 0.9$ - $\AA$ (for 291 Ca-pairs) and $\sim 0.8-\AA$ (289 Ca-pairs) for nsp16 and of $\sim 0.5-\AA \AA$ (109 Ca-pairs) and $\sim 0.8-\AA$ (110 Ca-pairs) for nsp10, respectively (Fig. 1b). Obvious conformational difference, however, was observed for the af/aZ interloop as well as the $\eta 3$-helix and its following loop (designated as n3-loop) in nsp16. In addition, the $a 1^{\prime}$-helix of nsp10 was completely densityuntraceable in our structure, which can be clearly observed in both SARS-CoV and MERS-CoV nsp10 (Fig. 1b).

Facilitated by the solved structure, we then characterized the key structural elements involved in the nsp10/nsp16 function. 1. Within the heterodimer complex, nsp10 is indispensable by acting as a co-factor for the nsp16 methylase. ${ }^{2,3}$ This intimate interaction is mediated by a total of 31 (N40, V42, K43, M44, L45, C46, T47, P59, G70, A71, C77, R78, K93, G94, and Y96 in nsp10 and K38, G39, 140, M41, V44, K76, V78, P80, A83, R86, Q87, V104, S105, D106, L244, and M247 in nsp16) residues, forming an extended interface with a buried surface area of $\sim 933 \AA^{2}$ and $\sim 872 \AA^{2}$ in nsp10 and nsp16, respectively (Fig. 1c). 2. Within nsp10, two zinc-fingers are observed to form to stabilize the nsp10 structure. One zinc ion is coordinated by residues $\mathrm{C} 74, \mathrm{C} 77, \mathrm{H} 83$, and $\mathrm{C} 90$, and the other by $\mathrm{C} 117, \mathrm{C} 120$, C128, and C130 (Fig. 1d). 3. Within nsp16, an SAM-binding pocket is constituted by amino acids N43, Y47, G71, G81, D99, N101, C115, and D130. These residues and the neighboring water molecules form a hydrogen-bond network to trap the bound SAM in an extended conformation (Fig. 1e). All these SAM-engaging residues are conserved in SARS-CoV and MERS-CoV, highlighting a conserved SAM-binding mode in these viruses (Supplementary Fig. S3a). The conserved SAM-interaction mode also highlights the possibility of developing a pan-antiviral inhibitors by targeting this SAM-binding pocket. 4. Within nsp16, a putative RNA-substrate binding groove is lined on one side by the af/aZ interloop and on the other by the n3-loop. These components have created an extended solvent-exposed groove with a strong positive electrostatic surface (Supplementary Fig. S4a). Notably, an intergroove bridge was observed to form between Y30 and K137 in our structure, which parallels our structure more to a recently-solved SARS-CoV-2 nsp10/nsp16 structure with a bound ${ }^{7 M e}$ GpppA cap (PDB: 6WKS) and the structure of MERS-CoV nsp10/nsp16 in complex with an RNA-cap substrate (PDB: 5YNM) than to the 
a

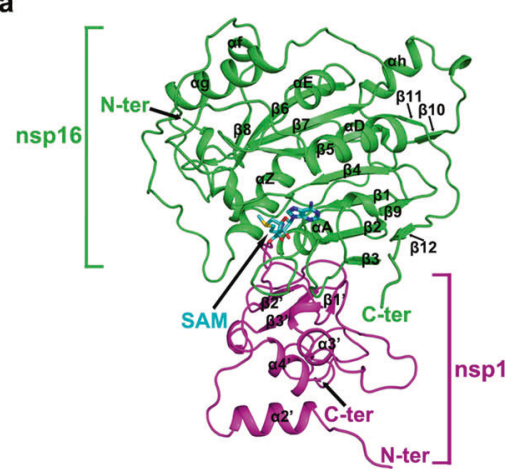

Overall structure

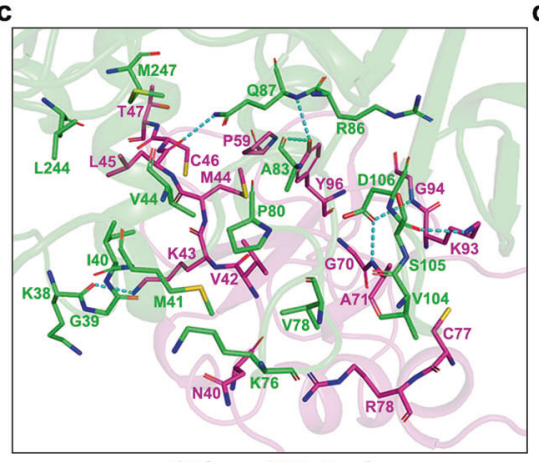

nsp10/nsp16 Interface

$\mathbf{f}$

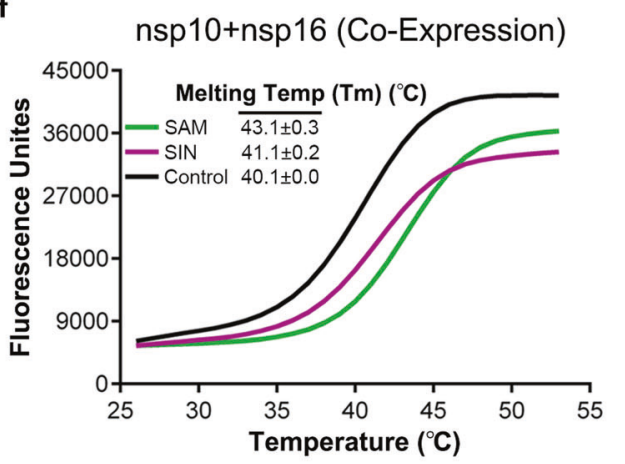

g

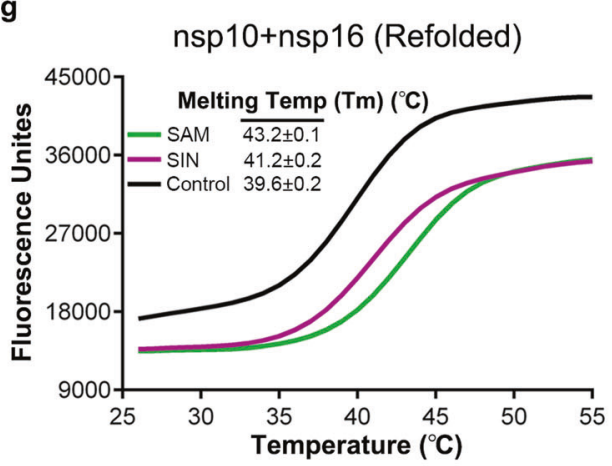

b

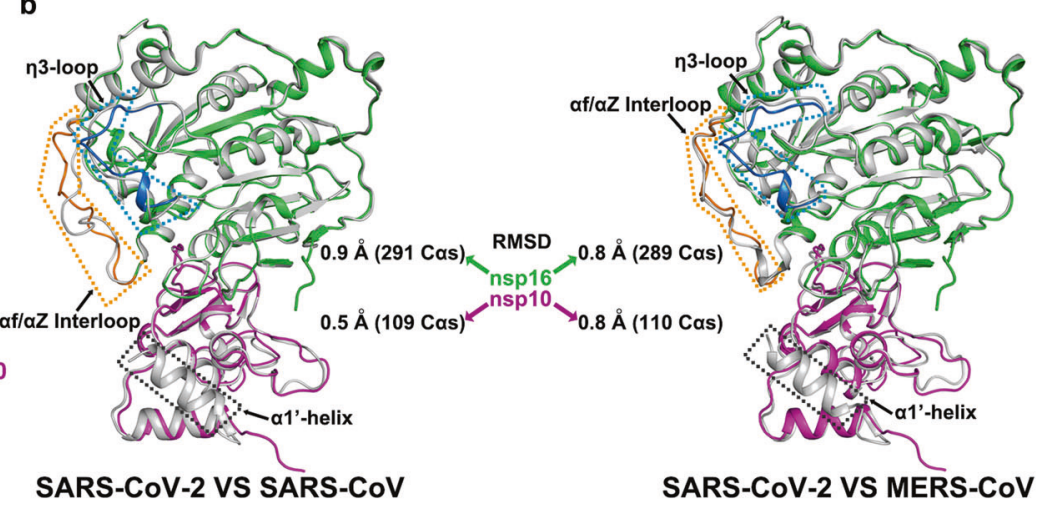

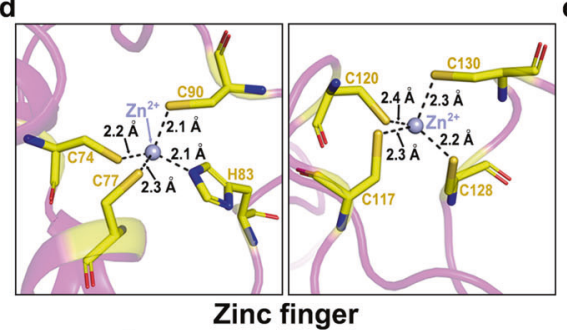

h
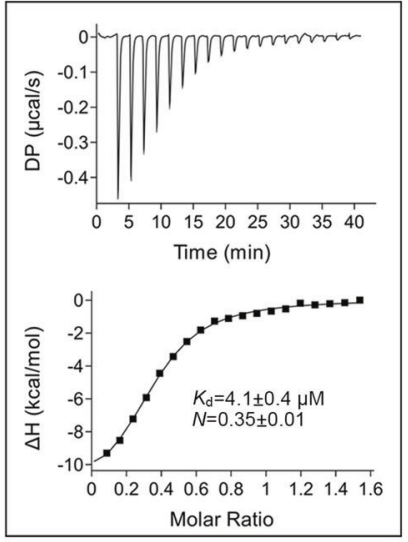

SAM to nsp10+nsp16 (Co-Expression) j
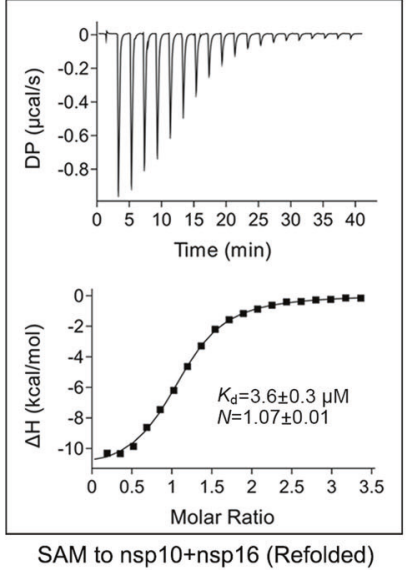

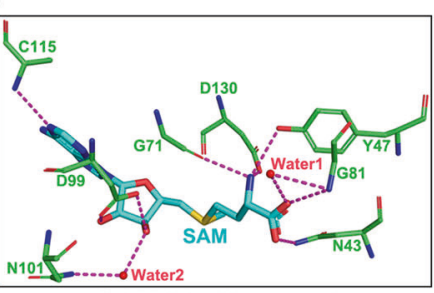

i

SAM binding pocket

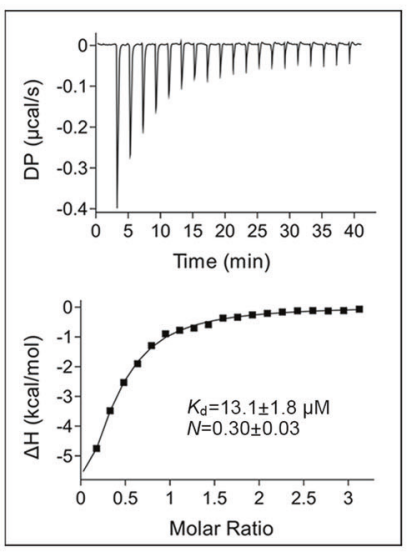
k

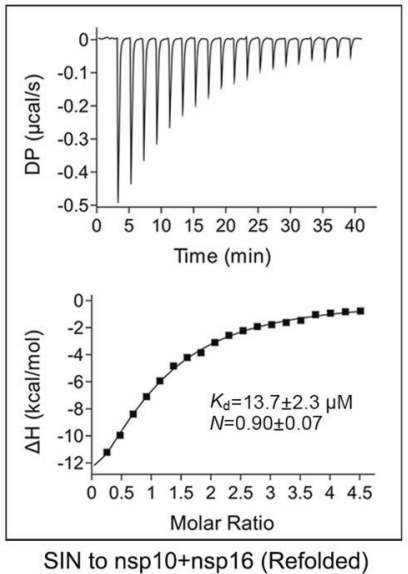

SARS-CoV nsp10/nsp16 structure (PDB: 3R24) whose RNA-binding groove is completely open (Supplementary Fig. S4a). This may indicate that our structure has accidentally trapped the groove in a post RNA binding conformation during crystallization, though the RNA-substrate is absent. The observations also highlight the plasticity of the RNA-binding groove in nsp16. Accordingly, previous studies on SARS-CoV nsp10/nsp16 indeed showed that the groove-lining residues are of high flexibility, showing either weak electron-densities or high B factors. ${ }^{2,3}$ It is also notable that a recent study reporting the SARS-CoV-2 nsp10/nsp16 structure in 
Fig. 1 Structure of SARS-CoV-2 nsp10/nsp16 hetero-dimer. a Overall structure of the hetero-complex formed between nsp10 (magenta) and nsp16 (green). The secondary structural elements and the bound SAM molecule are labeled. b Superimposition of the nsp10/nsp16 structure of SARS-CoV-2 onto those of SARS-CoV (left panel, PDB: 3R24) and MERS-CoV (right panel, PDB: 5YN5). The color scheme for our structure is the same as in a, and the SARS-CoV and MERS-CoV structures are shown in gray. Those elements exhibiting variant conformations are highlighted and marked. c Detailed interactions at the nsp10/nsp16 binding interface. Dashed lines indicate hydrogen-bonds. d A magnified view on the two zinc-finger motifs in nsp10. e Detailed interactions between SAM and nsp16 at the SAM-binding pocket. $\mathbf{f}$, $\mathbf{g}$ Interaction of SAM and SIN with SARS-CoV-2 nsp10/nsp16 characterized by differential scanning fluorimetry (DSF). The DSF data obtained with the proteincomplex purified directly from E. coli (designated as co-expression) and the protein-complex after the denaturing-refolding cycle (designated as refolded) are shown in $\mathbf{f}$ and $\mathbf{g}$, respectively. $\mathbf{h}-\mathbf{k}$ Binding of SAM and SIN to SARS-CoV-2 nsp10/nsp16 characterized by isothermal titration calorimetry (ITC). h SAM to nsp10/nsp16 (co-expression). i SIN to nsp10/nsp16 (co-expression). j SAM to nsp10/nsp16 (refolded). k SIN to nsp10/nsp16 (refolded)

the ternary state with bound SAM and ${ }^{7 M e} \mathrm{GpppA}$ has proposed a large conformational change associated with cap-binding. ${ }^{4}$ The fact that we have been able to trap the cap-binding groove in a post-RNA binding conformation without the bound RNA-cap substrate further suggests that the proposed conformational change is unlikely a substrate-binding induced event but rather the result of the intrinsic plasticity of the RNA-binding groove.

We finally investigated the interactions of SARS-CoV-2 nsp10/ nsp16 with SAM, sinefugin (SIN), and the ${ }^{7 M e}$ GpppA RNA-cap substrate in solution. Noted that nsp10/nsp16 likely has simultaneously captured SAM in its SAM-binding pocket during coexpression in E. coli, we further denatured the protein heterodimer to remove any residual SAM and then refolded the protein complex to obtain nsp10/nsp16 without pre-bound SAM (Supplementary Fig. S1b). The protein preparations (co-expression and refolded) were then used in parallel in the differential scanning fluorimetry (DSF) and isothermal titration calorimetry (ITC) assays. Potent interactions were expectedly observed for both SAM and SIN. The calculated shift in melting-temperature for SIN, however, was lower than that for SAM by $\sim 2^{\circ}$ (Fig. 1f, g). The ITC data further showed that SIN binds to SARS-CoV-2 nsp10/nsp16 $(13.1 \pm 1.8 \mu \mathrm{M}$ and $13.7 \pm 2.3 \mu \mathrm{M}$ to the co-expression and refolded proteins, respectively) with an over three-fold lower affinity than SAM $(4.1 \pm 0.4 \mu \mathrm{M}$ and $3.6 \pm 0.3 \mu \mathrm{M}$ to the co-expression and refolded proteins, respectively) (Fig. $1 \mathrm{~h}-\mathrm{k}$ ). It is also notable that the stoichiometry calculated with the protein purified directly from E. coli were $\sim 0.35$ for SAM and $\sim 0.3$ for SIN, indicating that about 2/ 3 of the SAM-binding pockets were pre-occupied during expression. These values were determined to be $\sim 1.07$ and $\sim 0.9$ with the refolded protein, well echoing our structural observation showing that the heterodimer contains a single SAM-binding pocket in nsp16 (Fig. 1h-k). The binding affinity between SARS-CoV-2 nsp10/ nsp16 and the ${ }^{7 M e}$ GpppA RNA-cap substrate was also investigated via ITC, which was determined to be $9.4 \pm 2.1 \mu \mathrm{M}$ (Supplementary Fig. S4b). Mutation of the bridging residues $\mathrm{Y} 30$ and K137 in nsp16 into Ala could abolish such binding. While the double mutation did not affect the solution gel-filtration behavior of the nsp10/nsp16 complex (Supplementary Fig. S1c), no direct interactions were detected in ITC for the Y30A/K137A mutant protein, demonstrating the important roles of the two amino acids in cap-recognition.

In conclusion, we have characterized the structural features of SARS-CoV-2 nsp10/nsp16 2'-O-methylase at the atomic level. The conserved SAM-binding pocket could be targeted by SAManalogs such as SIN. In addition, the plastic feature observed for the RNA-binding groove might provide new opportunities for antiviral drug design. Finally, the similar overall structures, conserved SAM-binding pockets, and resembled nsp10/nsp 16 binding interface shared by the nsp10/nsp16 2'-O-methylases of SARS-CoV-2, SARS-CoV, and MERS-CoV highlight that these enzymes are unlikely associated with the different pathogenic behaviors of these coronaviruses. The difference in the pathogenesis and transmission-capacity between SARS-CoV-2 and other coronaviruses should be related to the unique structural features and functional elements of other viral proteins. E.g., SARS-CoV-2 spike has been shown to possess an apparently higher affinity for human receptor ACE2 than that observed for SARS-CoV spike. ${ }^{5}$

\section{DATA AVAILABILITY}

The data sets used and/or analyzed during the current study are available from the corresponding author on reasonable request. Atomic coordinates and structure factors of the reported crystal structures have been deposited into the Protein Data Bank (https://www.rcsb.org; PDB: 7C2I, 7C2J).

\section{ACKNOWLEDGEMENTS}

We thank the staff of BL19U1 beamline at National Center for Protein Sciences Shanghai and Shanghai Synchrotron Radiation Facility (Shanghai, People's Republic of China) for assistance during data collection. This work was supported by the special research fund on COVID-19 of Sichuan Province (grant No. 2020YFS0010) and the special research fund on COVID-19 of West China Hospital Sichuan University (grant No. HX-2019-nCoV-004).

\section{AUTHOR CONTRIBUTIONS}

G.L. conceived the study and supervised the whole project. S.L. and H.C. conducted the majority of the experiments. S.L. and F.Y. collected the data-sets and solved the structures. F-L.Y., Z.C., Y.Z., and J.Q. facilitated in the DSF and ITC assays. G.L. and S.L. wrote the manuscript. S.Y. and Y.C. participated in the manuscript-editing and discussion.

\section{ADDITIONAL INFORMATION}

The online version of this article (https://doi.org/10.1038/s41392-020-00241-4) contains supplementary material, which is available to authorized users.

Competing interests: The authors declare no competing interests.

Sheng Lin ${ }^{1}$, Hua Chen ${ }^{1}$, Fei Ye ${ }^{1}$, Zimin Chen ${ }^{1}$, Fanli Yang ${ }^{1}$, Yue Zheng ${ }^{1}$, Yu Cao ${ }^{1,2}$, Jingxin Qiao ${ }^{1}$, Shengyong Yang ${ }^{1}$ and Guangwen $\mathrm{Lu}^{1}$

${ }^{1}$ West China Hospital Emergency Department (WCHED), State Key Laboratory of Biotherapy and Cancer Center, West China Hospital and Collaborative Innovation Center of Biotherapy, Sichuan University, 610041 Chengdu, Sichuan, China and ${ }^{2}$ Disaster Medicine Center, West China Hospital, Sichuan University, 610041 Chengdu, Sichuan, China

These authors contributed equally: Sheng Lin, Hua Chen Correspondence: Guangwen Lu (lugw@scu.edu.cn)

\section{REFERENCES}

1. WHO. Coronavirus disease (COVID-19) outbreak situation. https://www.who.int/ emergencies/diseases/novel-coronavirus-2019. Accessed 6 May 2020.

2. Chen, Y. et al. Biochemical and structural insights into the mechanisms of SARS coronavirus RNA ribose 2'-O-methylation by nsp $16 / \mathrm{nsp} 10$ protein complex. PLoS Pathog. 7, e1002294 (2011).

3. Decroly, E. et al. Crystal structure and functional analysis of the SARS-coronavirus RNA cap 2'-O-methyltransferase nsp10/nsp16 complex. PLoS Pathog. 7, e1002059 (2011) 
4. Viswanathan, T. et al. Structural basis of RNA cap modification by SARS-CoV-2 coronavirus. BioRxiv (2020).

5. Wang, Q. et al. Structural and functional basis of SARS-CoV-2 entry by using human ACE2. Cell 181, 894-904 (2020).

(c) (i) Open Access This article is licensed under a Creative Commons cc) Attribution 4.0 International License, which permits use, sharing, adaptation, distribution and reproduction in any medium or format, as long as you give appropriate credit to the original author(s) and the source, provide a link to the Creative
Commons license, and indicate if changes were made. The images or other third party material in this article are included in the article's Creative Commons license, unless indicated otherwise in a credit line to the material. If material is not included in the article's Creative Commons license and your intended use is not permitted by statutory regulation or exceeds the permitted use, you will need to obtain permission directly from the copyright holder. To view a copy of this license, visit http://creativecommons. org/licenses/by/4.0/.

(c) The Author(s) 2020 